\title{
Enumeration of weighted paths on a digraph and block hook determinant
}

https://doi.org/10.1515/spma-2020-0130

Received December 9, 2020; accepted March 18, 2021

Abstract: In this article, we evaluate determinants of "block hook" matrices, which are block matrices consist of hook matrices. In particular, we deduce that the determinant of a block hook matrix factorizes nicely. In addition we give a combinatorial interpretation of the aforesaid factorization property by counting weighted paths in a suitable weighted digraph.

Keywords: block hook matrix; determinants; weighted path; combinatorial proof

MSC: 05A10; 05A05; 11C20; 05C30; 05C38

\section{Introduction}

The evaluation of determinants is a nice topic, and fascinating for many people $[4,6,7,12,16]$. A huge amount of such evaluation has been collected in $[6,7]$. Specially, the problem of calculating the determinant of a $2 \times 2$ block matrix has been long studied [12, 16]. Block matrices are applied all over in mathematics and physics. They appear naturally in the description of systems with multiple discrete variables [13, 14]. Moreover, block matrices are utilized in many computational methods familiar to researchers of fluid dynamics [10]. Also the determinants of these matrices are found over a large number of area for both analytical and numerical applications $[8,11]$. The purpose of this paper is to evaluate the determinantal formulas of some special classes of block matrices, known as block hook matrix (defined later). In particular, we will show that the determinants of these block hook matrices admit nice product formulas. Now, let us define hook matrix in a precise way. First we need to define the following.

Definition 1.1. A square matrix is called an hook matrix if the pattern of the entries satisfy one of the following four conditions;

(a) all the entries right and below of the entry at the $(i, i)^{\text {th }}(i=1, \cdots, m)$ position are same

(b) all the entries left and above of the entry at the $(m+1-i, m+1-i)^{\text {th }}(i=1, \cdots, m)$ position are same

(c) all the entries right and above of the entry at the $(m+1-i, i)^{\text {th }}(i=1, \cdots m)$ position are same

(d) all the entries left and below of the entry at the $(i, m+1-i)^{\text {th }}(i=1, \cdots m)$ position are same.

Now consider the matrices in (1), (2), (3) and (4).

$$
A_{m}\left(x_{1}, \cdots, x_{m}\right)=\left(\begin{array}{ccccc}
x_{m} & x_{m} & \cdots & x_{m} & x_{m} \\
x_{m} & x_{m-1} & \cdots & x_{m-1} & x_{m-1} \\
\vdots & \vdots & \ddots & \vdots & \vdots \\
x_{m} & x_{m-1} & \cdots & x_{2} & x_{2} \\
x_{m} & x_{m-1} & \cdots & x_{2} & x_{1}
\end{array}\right)
$$

^Corresponding Author: Sudip Bera: Department of Mathematics, Indian Institute of Science, Bangalore 560 012, E-mail: sudipbera@iisc.ac.in 


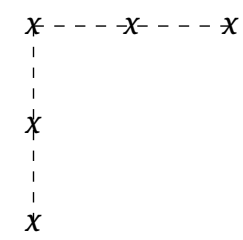

$\mathbf{H}_{\mathbf{A}}$

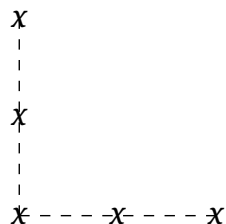

$\mathbf{H}_{\mathrm{C}}$

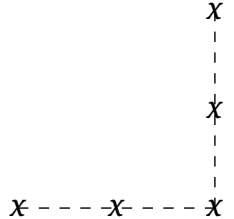

$\mathbf{H}_{\mathrm{B}}$

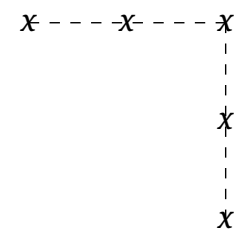

$\mathbf{H}_{\mathrm{D}}$

Figure 1: $H_{A}, H_{C}, H_{B}, H_{D}$ are four different shapes hook.

$$
\begin{aligned}
& B_{m}\left(x_{1}, \cdots, x_{m}\right)=\left(\begin{array}{ccccc}
x_{1} & x_{2} & \cdots & x_{m-1} & x_{m} \\
x_{2} & x_{2} & \cdots & x_{m-1} & x_{m} \\
\vdots & \vdots & \ddots & \vdots & \vdots \\
x_{m-1} & x_{m-1} & \cdots & x_{m-1} & x_{m} \\
x_{m} & x_{m} & \cdots & x_{m} & x_{m}
\end{array}\right) \\
& C_{m}\left(x_{1}, \cdots, x_{m}\right)=\left(\begin{array}{ccccc}
x_{m} & x_{m-1} & \cdots & x_{2} & x_{1} \\
x_{m} & x_{m-1} & \cdots & x_{2} & x_{2} \\
\vdots & \vdots & \ddots & \vdots & \vdots \\
x_{m} & x_{m-1} & \cdots & x_{m-1} & x_{m-1} \\
x_{m} & x_{m} & \cdots & x_{m} & x_{m}
\end{array}\right) \\
& D_{m}\left(x_{1}, \cdots, x_{m}\right)=\left(\begin{array}{ccccc}
x_{m} & x_{m} & \cdots & x_{m} & x_{m} \\
x_{m-1} & x_{m-1} & \cdots & x_{m-1} & x_{m} \\
\vdots & \vdots & \ddots & \vdots & \vdots \\
x_{2} & x_{2} & \cdots & x_{m-1} & x_{m} \\
x_{1} & x_{2} & \cdots & x_{m-1} & x_{m}
\end{array}\right)
\end{aligned}
$$

For each of the above matrix the pattern should be clear: In case of the matrix in (1), all the entries right and below of the entry at the $(i, i)^{\text {th }}(i=1, \cdots, m)$ position are same and clearly these entries form a hook of shape like $H_{A}$ in Figure 1. Similarly, for the matrix in (4), all the entries left and below of the entry at the $(i, m+1-i)^{\text {th }}(i=1, \cdots m)$ position are same and these entries form a hook of shape like $H_{D}$ in Figure 1 . Similarly the entries in the matrices in (2) and (3) form a hook of shape like $H_{B}$ and $H_{C}$ (in Figure 1) respectively. Moreover we have the following nice product formulas regarding the determinant of these hook matrices.

Proposition 1.1. Let $A_{m}\left(x_{1}, \cdots, x_{m}\right)$ be a matrix defined as (1). Then

$$
\operatorname{det}\left(A_{m}\left(x_{1}, \cdots, x_{m}\right)\right)=\prod_{i=1}^{m}\left(x_{i}-x_{i+1}\right), \text { where } x_{m+1}=0 \text {. }
$$

Proof. We apply the following row operations on the matrix $A_{m}\left(x_{1}, \cdots, x_{m}\right)$;

$$
R_{i}^{\prime}=R_{i}-R_{i-1} \text {, for } i=2, \cdots, m .
$$

Clearly, the resulting matrix (matrix obtained after row operations) is a diagonal matrix with diagonal entries $\left(x_{i}-x_{i+1}\right)$, for $i=m, m-1, \cdots, 1$, where $x_{m+1}=0$. Hence the result.

Proposition 1.2. Let $B_{m}\left(x_{1}, \cdots, x_{m}\right)$ be a matrix defined as (2). Then

$$
\operatorname{det} B_{m}\left(x_{1}, \cdots, x_{m}\right)=\prod_{i=1}^{m}\left(x_{i}-x_{i+1}\right), \text { where } x_{m+1}=0 .
$$


Proof. We apply the following row and column interchange on the matrix $A_{m}\left(x_{1}, \cdots, x_{m}\right)$;

$$
R_{i} \leftrightarrow R_{m-i+1} \text { and } C_{i} \leftrightarrow C_{m-i+1}, \text { for } i= \begin{cases}1,2, \cdots, \frac{m}{2}, & \text { if } m \text { is even } \\ 1,2, \cdots, \frac{m+1}{2}-1, & \text { if } m \text { is odd }\end{cases}
$$

Evidently the resulting matrix is $B_{m}\left(x_{1}, \cdots, x_{m}\right)$. This completes the proof.

Proposition 1.3. Let $C_{m}\left(x_{1}, \cdots, x_{m}\right)$ be a matrix defined as (3). Then

$$
\operatorname{det} C_{m}\left(x_{1}, \cdots, x_{m}\right)= \begin{cases}(-1)^{\frac{m}{2}} \times \prod_{i=1}^{m}\left(x_{i}-x_{i+1}\right), & \text { if } m \text { is even } \\ (-1)^{\frac{m-1}{2}} \times \prod_{i=1}^{m}\left(x_{i}-x_{i+1}\right), & \text { if } m \text { is odd },\end{cases}
$$

where $x_{m+1}=0$.

Proof. Here we apply the same row operations depicted as (5) on the matrix $A_{m}\left(x_{1}, \cdots, x_{m}\right)$, and as a result we get the matrix $C_{m}\left(x_{1}, \cdots, x_{m}\right)$. So the proposition.

Proposition 1.4. Let $D_{m}\left(x_{1}, \cdots, x_{m}\right)$ be a matrix defined as (4). Then

$$
\operatorname{det} D_{m}\left(x_{1}, \cdots, x_{m}\right)= \begin{cases}(-1)^{\frac{m}{2}} \times \prod_{i=1}^{m}\left(x_{i}-x_{i+1}\right), & \text { if } m \text { is even } \\ (-1)^{\frac{m-1}{2}} \times \prod_{i=1}^{m}\left(x_{i}-x_{i+1}\right), & \text { if } m \text { is odd, }\end{cases}
$$

where $x_{m+1}=0$.

Proof. Applying the same column operations described as (5) on the matrix $A_{m}\left(x_{1}, \cdots, x_{m}\right)$, we get the matrix $D_{m}\left(x_{1}, \cdots, x_{m}\right)$. Hence the proposition.

Definition 1.2. An $N \times N(N \geq 2)$ block matrix $M$ is called a block hook matrix if each block of $M$ is a hook matrix.

Remark 1. If $N=1$, then the block hook matrix reduces to a hook matrix.

\section{Example 1.5.}

$$
\left(\begin{array}{cc|cc}
x_{2} & x_{2} & y_{2} & y_{2} \\
x_{2} & x_{1} & y_{2} & y_{1} \\
\hline z_{2} & z_{2} & w_{2} & w_{2} \\
z_{2} & z_{1} & w_{2} & w_{1}
\end{array}\right),\left(\begin{array}{cc|cc}
x_{1} & x_{2} & y_{2} & y_{1} \\
x_{2} & x_{2} & y_{2} & y_{2} \\
\hline z_{1} & z_{2} & w_{2} & w_{1} \\
z_{2} & z_{2} & w_{2} & w_{2}
\end{array}\right),\left(\begin{array}{cc|cc}
x_{1} & x_{2} & y_{2} & y_{1} \\
x_{2} & x_{2} & y_{2} & y_{2} \\
\hline z_{2} & z_{2} & w_{2} & w_{2} \\
z_{1} & z_{2} & w_{2} & w_{1}
\end{array}\right),\left(\begin{array}{cc|cc}
x_{2} & x_{2} & y_{2} & y_{2} \\
x_{2} & x_{1} & y_{1} & y_{2} \\
\hline z_{2} & z_{2} & w_{2} & w_{2} \\
z_{2} & z_{1} & w_{1} & w_{2}
\end{array}\right) .
$$

Example 1.5 contains four $2 \times 2$ block matrices, in which each block is a hook. In fact, the $1^{\text {st }}$ matrix contains four hooks of shape $H_{A}$ as depicted in Figure 1, whereas the hooks in the last matrix are of different shapes. Also it can be shown that, the determinant of any one of the above matrices gives a nice product formula. So, one natural question occurs: Is it true that the determinant of any block hook matrix admits such a product formula? In this paper, we formulate different classes of block hook matrices of order $N m(N \times N$ block matrix and order of each block is $m$ ) and prove that the determinants of all these block hook matrices can be written as $\prod_{i=1}^{m} \operatorname{det}\left(X_{(i, i+1)}\right)$ (upto sign), where

$$
X_{(i, i+1)}=\left(\begin{array}{cccc}
x_{i}^{(1,1)}-x_{i+1}^{(1,1)} & x_{i}^{(1,2)}-x_{i+1}^{(1,2)} & \cdots & x_{i}^{(1, N)}-x_{i+1}^{(1, N)} \\
x_{i}^{(2,1)}-x_{i+1}^{(2,1)} & x_{i}^{(2,2)}-x_{i+1}^{(2,2)} & \cdots & x_{i}^{(2, N)}-x_{i+1}^{(2, N)} \\
\vdots & \vdots & \ddots & \vdots \\
x_{i}^{(N, 1)}-x_{i+1}^{(N, 1)} & x_{i}^{(N, 2)}-x_{i+1}^{(N, 2)} & \cdots & x_{i}^{(N, N)}-x_{i+1}^{(N, N)}
\end{array}\right)
$$


Moreover, we give combinatorial explanation of these product formulas. Throughout this paper we denote $i \times j$ zero matrix by $O_{i, j}, R_{i} \leftrightarrow R_{j}\left(C_{i} \leftrightarrow C_{j}\right)$ denotes the row (column) interchange between $i^{\text {th }}$ and $j^{\text {th }}$ row (column) of a matrix, we denote the set $\{1,2, \cdots, m\}$ by $[m]$.

Now, let us briefly summarize the content. In Section 2, we consider block hook matrices containing same shape hooks and derive the product formulas for the determinants of these matrices. In Section 3, we focus on the block hook matrices formed by hooks of two different shapes and we show that the determinant of these matrices admit nice product formulas. In Section 4, we deal with block hook matrices containing hooks of four different shapes and find similar product formulas. In Section 5, we give combinatorial interpretations of factorization property of block hook determinants.

\section{Block matrices containing hooks of same shape}

In this section, we evaluate the determinants of block hook matrices, which are block matrices, in which each block is a hook and shape of all hooks are same. Now, we introduce an $N \times N$ block matrix $A(N, m)$ in the following way;

$$
A(N, m)=\left(A_{i j}\right) \text {, where } 1 \leq i, j \leq N \text { and each block } A_{i j}=A_{m}\left(x_{1}^{(i, j)}, \cdots, x_{m}^{(i, j)}\right) .
$$

So $A(N, m)$ is a hook matrix of order $N m$. For example,

$$
A(3,2)=\left(\begin{array}{cc|cc|cc}
x_{2}^{(1,1)} & x_{2}^{(1,1)} & x_{2}^{(1,2)} & x_{2}^{(1,2)} & x_{2}^{(1,3)} & x_{2}^{(1,3)} \\
x_{2}^{(1,1)} & x_{1}^{(1,1)} & x_{2}^{(1,2)} & x_{1}^{(1,2)} & x_{2}^{(1,3)} & x_{1}^{(1,3)} \\
\hline x_{2}^{(2,1)} & x_{2}^{(2,1)} & x_{2}^{(2,2)} & x_{2}^{(2,2)} & x_{2}^{(2,3)} & x_{2}^{(2,3)} \\
x_{2}^{(2,1)} & x_{1}^{(2,1)} & x_{2}^{(2,2)} & x_{1}^{(2,2)} & x_{2}^{(2,3)} & x_{1}^{(2,3)} \\
\hline x_{2}^{(3,1)} & x_{2}^{(3,1)} & x_{2}^{(3,2)} & x_{2}^{(3,2)} & x_{2}^{(3,3)} & x_{2}^{(3,3)} \\
x_{2}^{(3,1)} & x_{1}^{(3,1)} & x_{2}^{(3,2)} & x_{1}^{(3,2)} & x_{2}^{(3,3)} & x_{1}^{(3,3)}
\end{array}\right)
$$

is a block hook matrix, whose each block is a hook like (1).

Theorem 2.1. Let $A(N, m)$ be a block hook matrix of order Nm defined as (7). Then

$$
\operatorname{det}(A(N, m))=\prod_{i=1}^{m} \operatorname{det}\left(X_{(i, i+1)}\right),
$$

where $X_{(i, i+1)}$ is defined as (6) and $x_{m+1}^{(i, j)}=0$, for all $1 \leq i, j \leq N$.

Proof. We prove the theorem by applying induction on $m$. For $m=1$, clearly the matrix

$$
A(N, 1)=\left(\begin{array}{c|c|c|c}
x_{1}^{(1,1)} & x_{1}^{(1,2)} & \cdots & x_{1}^{(1, N)} \\
\hline x_{1}^{(2,1)} & x_{1}^{(2,2)} & \cdots & x_{1}^{(2, N)} \\
\hline \vdots & \vdots & \ddots & \vdots \\
\hline x_{1}^{(N, 1)} & x_{1}^{(N, 2)} & \cdots & x_{1}^{(N, N)}
\end{array}\right)=X_{(1,2)} \text {, with } x_{2}^{(i, j)}=0 \text {, for all } 1 \leq i, j \leq N .
$$

So, the base case is true. Suppose the result is true for all such matrices $A(N,(m-1))$. Now to complete the proof we want to go through the following row operations on the matrix $A(N, m)$;

$$
R_{i}^{\prime}=R_{i}-R_{i-1} \text {, for } i=m, 2 m, \cdots, N m .
$$


Then the matrix, we obtain thereby is as follows:

All the rows of each block $A_{i j}$ remain unchanged except the last row. The last row of each block $A_{i j}$ looks like $0,0, \cdots,\left(x_{1}^{(i, j)}-x_{2}^{(i, j)}\right)$. So for $\ell=1,2, \cdots, N$, the $(\ell m)^{\text {th }}$ row of the matrix $A(N, m)$ is

$$
0,0, \cdots,\left(x_{1}^{(\ell, 1)}-x_{2}^{(\ell, 1)}\right), 0,0, \cdots,\left(x_{1}^{(\ell, 2)}-x_{2}^{(\ell, 2)}\right), \cdots, 0,0, \cdots,\left(x_{1}^{(\ell, N)}-x_{2}^{(\ell, N)}\right) .
$$

Now we apply the successive row interchange on the resulting matrix (after performing row operations on the matrix $A(N, m))$ to arrange the rows in the following order;

$$
R_{m}, R_{2 m}, \cdots, R_{N m}, R_{1}, \cdots, R_{m-1}, R_{m+1}, \cdots, R_{2 m-1}, \cdots, R_{(N-1) m+1}, \cdots, R_{N m-1} .
$$

Then by successive column interchange we arrange the columns in the following order;

$$
C_{m}, C_{2 m}, \cdots, C_{N m}, C_{1}, \cdots, C_{m-1}, C_{m+1}, \cdots, C_{2 m-1}, \cdots, C_{(N-1) m+1}, \cdots, C_{N m-1} .
$$

And finally we obtain the matrix of the form

$$
\left(\begin{array}{c|c}
X_{(1,2)} & O_{N,(N m-N)} \\
\hline \star & A(N,(m-1))
\end{array}\right),
$$

where $A(N,(m-1))$ is block matrix and each block is $A_{m-1}\left(x_{2}^{(i, j)}, \cdots, x_{m}^{(i, j)}\right)$. Therefore, $A(N,(m-1))$ is a block hook matrix of order $N(m-1)$. Now, without doubt we can write

$$
\operatorname{det}(A(N, m))=\left(\begin{array}{c|c}
X_{(1,2)} & O_{N,(N m-N)} \\
\hline \star & A(N,(m-1))
\end{array}\right) .
$$

Again by the Laplace expansion

$$
\operatorname{det}\left(\begin{array}{c|c}
X_{(1,2)} & O_{N,(N m-N)} \\
\hline \star & A(N,(m-1))
\end{array}\right)=\operatorname{det}\left(X_{(1,2)}\right) \times \operatorname{det}(A(N,(m-1))) .
$$

Now we set $x_{k}^{(i, j)}=y_{k-1}^{(i, j)}$, for $k=2,3, \cdots, m$ and $1 \leq i, j \leq N$. Then $A_{m-1}\left(x_{2}^{(i, j)}, \cdots, x_{m}^{(i, j)}\right)=$ $A_{m-1}\left(y_{1}^{(i, j)}, \cdots, y_{m-1}^{(i, j)}\right)$. Again by inductive hypothesis we can write

$$
\operatorname{det}(A(N,(m-1)))=\prod_{i=1}^{m-1} \operatorname{det}\left(Y_{(i, i+1)}\right)
$$

where $Y_{(i, i+1)}$ is the matrix obtained by putting $x_{i+1}^{(r, s)}-x_{i+2}^{(r, s)}=y_{i}^{(r, s)}-y_{i+1}^{(r, s)}$ in $X_{(i+1, i+2)}$ for all $1 \leq r, s \leq N$, and $y_{m}^{(i, j)}=x_{m+1}^{(i, j)}=0$. Clearly

$$
\prod_{i=1}^{m-1} \operatorname{det}\left(Y_{(i, i+1)}\right)=\prod_{i=2}^{m} \operatorname{det}\left(X_{(i, i+1)}\right)
$$

Therefore, by (9), (10), (11) and (12) we get

$$
\operatorname{det}(A(N, m))=\prod_{i=1}^{m} \operatorname{det}\left(X_{(i, i+1)}\right) .
$$

Here we think about the determinantal formulas for the block hook matrix formed by the hook matrices like $B_{m}\left(x_{1}, \cdots, x_{m}\right)$. Now we define an $N \times N$ block matrix $B(N, m)$ in the following way;

$$
B(N, m)=\left(B_{i j}\right) \text {, where } 1 \leq i, j \leq N \text { and } B_{i j}=B_{m}\left(x_{1}^{(i, j)}, \cdots, x_{m}^{(i, j)}\right) .
$$


So $B(N, m)$ is a block hook matrix of order $N m$. For example,

$$
B(3,2)=\left(\begin{array}{cc|cc|cc}
x_{1}^{(1,1)} & x_{2}^{(1,1)} & x_{1}^{(1,2)} & x_{2}^{(1,2)} & x_{1}^{(1,3)} & x_{2}^{(1,3)} \\
x_{2}^{(1,1)} & x_{2}^{(1,1)} & x_{2}^{(1,2)} & x_{2}^{(1,2)} & x_{2}^{(1,3)} & x_{2}^{(1,3)} \\
\hline x_{1}^{(2,1)} & x_{2}^{(2,1)} & x_{1}^{(2,2)} & x_{2}^{(2,2)} & x_{1}^{(2,3)} & x_{2}^{(2,3)} \\
x_{2}^{(2,1)} & x_{2}^{(2,1)} & x_{2}^{(2,2)} & x_{2}^{(2,2)} & x_{2}^{(2,3)} & x_{2}^{(2,3)} \\
\hline x_{1}^{(3,1)} & x_{2}^{(3,1)} & x_{1}^{(3,2)} & x_{2}^{(3,2)} & x_{1}^{(3,3)} & x_{2}^{(3,3)} \\
x_{2}^{(3,1)} & x_{2}^{(3,1)} & x_{2}^{(3,2)} & x_{2}^{(3,2)} & x_{2}^{(3,3)} & x_{2}^{(3,3)}
\end{array}\right) .
$$

Theorem 2.2. Let $B(N, m)$ be a block hook matrix of order $N m$ defined as (13). Then

$$
\operatorname{det}(B(N, m))=\prod_{i=1}^{m} \operatorname{det}\left(X_{(i, i+1)}\right)
$$

where $X_{(i, i+1)}$ is defined by (6) and $x_{m+1}^{(i, j)}=0$, for all $1 \leq i, j \leq N$.

Proof. We prove this theorem by performing some row and column operations on $A(N, m)$, so that the resulting matrix is $B(N, m)$. In fact, we perform the following row and column operations;

$$
\begin{aligned}
& R_{k m+i} \leftrightarrow R_{k m+(m-i+1)} \text { and } C_{k m+i} \leftrightarrow C_{k m+(m-i+1)}, \text { where } k=0,1, \cdots,(N-1) \text { and } \\
& \qquad i= \begin{cases}1,2, \cdots, \frac{m}{2}, & \text { if } m \text { is even } \\
1,2, \cdots, \frac{m+1}{2}-1, & \text { if } m \text { is odd } .\end{cases}
\end{aligned}
$$

Obviously, the resulting matrix is $B(N, m)$. Hence the result.

Now we evaluate the determinant of the block hook matrix $C(N, m)$ formed by the hook matrices of the form $C_{m}\left(x_{1}, \cdots, x_{m}\right)$. Let us define the matrix $C(N, m)$ as follows:

$$
C(N, m)=\left(C_{i j}\right) \text {, where } C_{i j}=C_{m}\left(x_{1}^{(i, j)}, \cdots, x_{m}^{(i, j)}\right) \text { and } 1 \leq i, j \leq N .
$$

For example,

$$
C(3,2)=\left(\begin{array}{cc|cc|cc}
x_{2}^{(1,1)} & x_{1}^{(1,1)} & x_{2}^{(1,2)} & x_{1}^{(1,2)} & x_{2}^{(1,3)} & x_{1}^{(1,3)} \\
x_{2}^{(1,1)} & x_{2}^{(1,1)} & x_{2}^{(1,2)} & x_{2}^{(1,2)} & x_{2}^{(1,3)} & x_{2}^{(1,3)} \\
\hline x_{2}^{(2,1)} & x_{1}^{(2,1)} & x_{2}^{(2,2)} & x_{1}^{(2,2)} & x_{2}^{(2,3)} & x_{1}^{(2,3)} \\
x_{2}^{(2,1)} & x_{2}^{(2,1)} & x_{2}^{(2,2)} & x_{2}^{(2,2)} & x_{2}^{(2,3)} & x_{2}^{(2,3)} \\
\hline x_{2}^{(3,1)} & x_{1}^{(3,1)} & x_{2}^{(3,2)} & x_{1}^{(3,2)} & x_{2}^{(3,3)} & x_{1}^{(3,3)} \\
x_{2}^{(3,1)} & x_{2}^{(3,1)} & x_{2}^{(3,2)} & x_{2}^{(3,2)} & x_{2}^{(3,3)} & x_{2}^{(3,3)}
\end{array}\right) .
$$

Theorem 2.3. Let $C(N, m)$ be a block hook matrix of order Nm defined as (17). Then

$$
\operatorname{det}(C(N, m))= \begin{cases}(-1)^{\frac{N m}{2}} \times \prod_{i=1}^{m} \operatorname{det}\left(X_{(i, i+1)}\right), & \text { if } m \text { is even } \\ (-1)^{\frac{N(m-1)}{2}} \times \prod_{i=1}^{m} \operatorname{det}\left(X_{(i, i+1)}\right), & \text { if } m \text { is odd }\end{cases}
$$

where $X_{(i, i+1)}$ is defined by (6) and $x_{m+1}^{(i, j)}=0$ for all $1 \leq i, j \leq N$. 
Proof. If we apply the same row operations on the matrix $A(N, m)$ as in the proof of Theorem 2.2, we get the matrix $C(N, m)$. Hence the theorem.

In this place we want to establish the determinantal formula for the block hook matrix $D(N, m)$, where $D(N, m)$ is an $N m$ ordered matrix defined as follows:

$$
\text { Now, } D(N, m)=\left(D_{i j}\right) \text {, where } D_{i j}=D_{m}\left(x_{1}^{(i, j)}, \cdots, x_{m}^{(i, j)}\right) \text { and } 1 \leq i, j \leq N \text {. }
$$

For example,

$$
D(3,2)=\left(\begin{array}{cc|cc|cc}
x_{2}^{(1,1)} & x_{2}^{(1,1)} & x_{2}^{(1,2)} & x_{2}^{(1,2)} & x_{2}^{(1,3)} & x_{2}^{(1,3)} \\
x_{1}^{(1,1)} & x_{2}^{(1,1)} & x_{1}^{(1,2)} & x_{2}^{(1,2)} & x_{1}^{(1,3)} & x_{2}^{(1,3)} \\
\hline x_{2}^{(2,1)} & x_{2}^{(2,1)} & x_{2}^{(2,2)} & x_{2}^{(2,2)} & x_{2}^{(2,3)} & x_{2}^{(2,3)} \\
x_{1}^{(2,1)} & x_{2}^{(2,1)} & x_{1}^{(2,2)} & x_{2}^{(2,2)} & x_{1}^{(2,3)} & x_{2}^{(2,3)} \\
\hline x_{2}^{(3,1)} & x_{2}^{(3,1)} & x_{2}^{(3,2)} & x_{2}^{(3,2)} & x_{2}^{(3,3)} & x_{2}^{(3,3)} \\
x_{1}^{(3,1)} & x_{2}^{(3,1)} & x_{1}^{(3,2)} & x_{2}^{(3,2)} & x_{1}^{(3,3)} & x_{2}^{(3,3)}
\end{array}\right) .
$$

Theorem 2.4. Let $D(N, m)$ be a block hook matrix of order Nm defined as (19). Then

$$
\operatorname{det}(D(N, m))= \begin{cases}(-1)^{\frac{N m}{2}} \times \prod_{i=1}^{m} \operatorname{det}\left(X_{(i, i+1)}\right), & \text { if } m \text { is even } \\ (-1)^{\frac{N(m-1)}{2}} \times \prod_{i=1}^{m} \operatorname{det}\left(X_{(i, i+1)}\right), & \text { if } m \text { is odd }\end{cases}
$$

where $X_{(i, i+1)}$ is defined by (6) and $x_{m+1}^{(i, j)}=0$ for all $1 \leq i, j \leq N$.

Proof. Applying the same column operations as in the proof of Theorem 2.2 on the matrix $A(N, m)$, we get the matrix $D(N, m)$. This proves the theorem.

\section{Block matrices containing hooks of two different shapes}

In this section, we present determinantal formulas for the block hook matrices containing hooks of two different shapes. In fact, we construct four different such block matrices and evaluate their determinants. Let $E(N, m)$ be a block hook matrix defined in the following way;

$$
E(N, m)=\left(E_{i j}\right)_{N \times N}, \text { where } E_{i j}= \begin{cases}C_{m}\left(x_{1}^{(i, j)}, \cdots, x_{m}^{(i, j)}\right), & \text { if } i \text { is even } \\ A_{m}\left(x_{1}^{(i, j)}, \cdots, x_{m}^{(i, j)}\right), & \text { if } i \text { is odd } .\end{cases}
$$

For example,

$$
E(3,2)=\left(\begin{array}{cc|cc|cc}
x_{2}^{(1,1)} & x_{2}^{(1,1)} & x_{2}^{(1,2)} & x_{2}^{(1,2)} & x_{2}^{(1,3)} & x_{2}^{(1,3)} \\
x_{2}^{(1,1)} & x_{1}^{(1,1)} & x_{2}^{(1,2)} & x_{1}^{(1,2)} & x_{2}^{(1,3)} & x_{1}^{(1,3)} \\
\hline x_{2}^{(2,1)} & x_{1}^{(2,1)} & x_{2}^{(2,2)} & x_{1}^{(2,2)} & x_{2}^{(2,3)} & x_{1}^{(2,3)} \\
x_{2}^{(2,1)} & x_{2}^{(2,1)} & x_{2}^{(2,2)} & x_{2}^{(2,2)} & x_{2}^{(2,3)} & x_{2}^{(2,3)} \\
\hline x_{2}^{(3,1)} & x_{2}^{(3,1)} & x_{2}^{(3,2)} & x_{2}^{(3,2)} & x_{2}^{(3,3)} & x_{2}^{(3,3)} \\
x_{2}^{(3,1)} & x_{1}^{(3,1)} & x_{2}^{(3,2)} & x_{1}^{(3,2)} & x_{2}^{(3,3)} & x_{1}^{(3,3)}
\end{array}\right) .
$$


Theorem 3.1. Let $E(N, m)$ be a block hook matrix of order $N m$ defined as (21). Then

$$
\operatorname{det}(E(N, m))= \begin{cases}(-1)^{\frac{N m}{4}} \prod_{i=1}^{m} \operatorname{det}\left(X_{(i, i+1)}\right), & \text { if } N \text { is even, } m \text { is even } \\ (-1)^{\frac{N(m-1)}{4}} \prod_{i=1}^{m} \operatorname{det}\left(X_{(i, i+1)}\right), & \text { if } N \text { is even, } m \text { is odd } \\ (-1)^{\frac{(N-1) m}{4}} \prod_{i=1}^{m} \operatorname{det}\left(X_{(i, i+1)}\right), & \text { if } N \text { is odd, } m \text { is even } \\ (-1)^{\frac{(N-1)(m-1)}{4}} \prod_{i=1}^{m} \operatorname{det}\left(X_{(i, i+1)}\right), & \text { if } N \text { is odd, } m \text { is odd }\end{cases}
$$

where $X_{(i, i+1)}$ is defined as (6) and $x_{m+1}^{(i, j)}=0$ for all $1 \leq i, j \leq N$.

Proof. Here we apply the following elementary row operations on the matrix $A(N, m)$;

$$
\begin{gathered}
R_{k m+i} \leftrightarrow R_{k m+(m-i+1)}, \text { where } \\
i=\left\{\begin{array}{ll}
1,2, \cdots, \frac{m}{2}, & \text { if } m \text { is even } \\
1,2, \cdots, \frac{m+1}{2}-1, & \text { if } m \text { is odd }
\end{array} \text { and } k= \begin{cases}1,3, \cdots, N-1, & \text { if } N \text { is even } \\
1,3, \cdots, N-2, & \text { if } N \text { is odd . }\end{cases} \right.
\end{gathered}
$$

Evidently the resulting matrix is $E(N, m)$. Hence the theorem.

Let us introduce a block hook matrix $E^{\prime}(N, m)$ in the following way;

$$
E^{\prime}(N, m)=\left(E_{i j}^{\prime}\right)_{N \times N}, \text { where } E_{i j}^{\prime}= \begin{cases}C_{m}\left(x_{1}^{(i, j)}, \cdots, x_{m}^{(i, j)}\right), & \text { if } i \text { is odd } \\ A_{m}\left(x_{1}^{(i, j)}, \cdots, x_{m}^{(i, j)}\right), & \text { if } i \text { is even }\end{cases}
$$

For example,

$$
E^{\prime}(3,2)=\left(\begin{array}{cc|cc|cc}
x_{2}^{(1,1)} & x_{1}^{(1,1)} & x_{2}^{(1,2)} & x_{1}^{(1,2)} & x_{2}^{(1,3)} & x_{1}^{(1,3)} \\
x_{2}^{(1,1)} & x_{2}^{(1,1)} & x_{2}^{(1,2)} & x_{2}^{(1,2)} & x_{2}^{(1,3)} & x_{2}^{(1,3)} \\
\hline x_{2}^{(2,1)} & x_{2}^{(2,1)} & x_{2}^{(2,2)} & x_{2}^{(2,2)} & x_{2}^{(2,3)} & x_{2}^{(2,3)} \\
x_{2}^{(2,1)} & x_{1}^{(2,1)} & x_{2}^{(2,2)} & x_{1}^{(2,2)} & x_{2}^{(2,3)} & x_{1}^{(2,3)} \\
\hline x_{2}^{(3,1)} & x_{1}^{(3,1)} & x_{2}^{(3,2)} & x_{1}^{(3,2)} & x_{2}^{(3,3)} & x_{1}^{(3,3)} \\
x_{2}^{(3,1)} & x_{2}^{(3,1)} & x_{2}^{(3,2)} & x_{2}^{(3,2)} & x_{2}^{(3,3)} & x_{2}^{(3,3)}
\end{array}\right) .
$$

Theorem 3.2. Let $E^{\prime}(N, m)$ be a block hook matrix of order $N m$ defined as (25). Then

$$
\operatorname{det}\left(E^{\prime}(N, m)\right)= \begin{cases}(-1)^{\frac{N m}{4}} \prod_{i=1}^{m} \operatorname{det}\left(X_{(i, i+1)}\right), & \text { if } N \text { is even, } m \text { is even } \\ (-1)^{\frac{N(m-1)}{4}} \prod_{i=1}^{m} \operatorname{det}\left(X_{(i, i+1)}\right), & \text { if } N \text { is even, } m \text { is odd } \\ (-1)^{\frac{(N-1) m}{4}} \prod_{i=1}^{m} \operatorname{det}\left(X_{(i, i+1)}\right), & \text { if } N \text { is odd, } m \text { is even } \\ (-1)^{\frac{(N-1)(m-1)}{4}} \prod_{i=1}^{m} \operatorname{det}\left(X_{(i, i+1)}\right), & \text { if } N \text { is odd, } m \text { is odd }\end{cases}
$$

where $X_{(i, i+1)}$ is defined as (6) and $x_{m+1}^{(i, j)}=0$ for all $1 \leq i, j \leq N$.

Proof. Applying the same row operations described by (23) and (24) on the matrix $C(N, m)$, we get the matrix $E^{\prime}(N, m)$. This completes the proof. 
Here, we define another block hook matrix $F(N, m)$ as follows:

$$
F(N, m)=\left(F_{i j}\right)_{N \times N}, \text { where } F_{i j}= \begin{cases}B_{m}\left(x_{1}^{(i, j)}, \cdots, x_{m}^{(i, j)}\right), & \text { if } i \text { is odd } \\ D_{m}\left(x_{1}^{(i, j)}, \cdots, x_{m}^{(i, j)}\right), & \text { if } i \text { is even }\end{cases}
$$

For example:

$$
F(3,2)=\left(\begin{array}{cc|cc|cc}
x_{1}^{(1,1)} & x_{2}^{(1,1)} & x_{1}^{(1,2)} & x_{2}^{(1,2)} & x_{1}^{(1,3)} & x_{2}^{(1,3)} \\
x_{2}^{(1,1)} & x_{2}^{(1,1)} & x_{2}^{(1,2)} & x_{2}^{(1,2)} & x_{2}^{(1,3)} & x_{2}^{(1,3)} \\
\hline x_{2}^{(2,1)} & x_{2}^{(2,1)} & x_{2}^{(2,2)} & x_{2}^{(2,2)} & x_{2}^{(2,3)} & x_{2}^{(2,3)} \\
x_{1}^{(2,1)} & x_{2}^{(2,1)} & x_{1}^{(2,2)} & x_{2}^{(2,2)} & x_{1}^{(2,3)} & x_{2}^{(2,3)} \\
\hline x_{1}^{(3,1)} & x_{2}^{(3,1)} & x_{1}^{(3,2)} & x_{2}^{(3,2)} & x_{1}^{(3,3)} & x_{2}^{(3,3)} \\
x_{2}^{(3,1)} & x_{2}^{(3,1)} & x_{2}^{(3,2)} & x_{2}^{(3,2)} & x_{2}^{(3,3)} & x_{2}^{(3,3)}
\end{array}\right) .
$$

Theorem 3.3. Let $F(N, m)$ be a block hook matrix of order Nm defined as (27). Then

$$
\operatorname{det}(F(N, m))= \begin{cases}(-1)^{\frac{N m}{4}} \prod_{i=1}^{m} \operatorname{det}\left(X_{(i, i+1)}\right), & \text { if } N \text { is even, } m \text { is even } \\ (-1)^{\frac{N(m-1)}{4}} \prod_{i=1}^{m} \operatorname{det}\left(X_{(i, i+1)}\right), & \text { if } N \text { is even, } m \text { is odd } \\ (-1)^{\frac{(N-1) m}{4}} \prod_{i=1}^{m} \operatorname{det}\left(X_{(i, i+1)}\right), & \text { if } N \text { is odd, } m \text { is even } \\ (-1)^{\frac{(N-1)(m-1)}{4}} \prod_{i=1}^{m} \operatorname{det}\left(X_{(i, i+1)}\right), & \text { if } N \text { is odd, } m \text { is odd }\end{cases}
$$

where $X_{(i, i+1)}$ is defined as (6) and $x_{m+1}^{(i, j)}=0$ for all $1 \leq i, j \leq N$.

Proof. Here we apply the same row operations described by (23) and (24) on the matrix $B(N, m)$. Evidently the resulting matrix is $F(N, m)$. Hence the theorem.

Let us define another block hook matrix $F^{\prime}(N, m)$ as follows:

$$
F^{\prime}(N, m)=\left(F_{i j}^{\prime}\right)_{N \times N}, \text { where } F_{i j}^{\prime}= \begin{cases}D_{m}\left(x_{1}^{(i, j)}, \cdots, x_{m}^{(i, j)}\right), & \text { if } i \text { is odd } \\ B_{m}\left(x_{1}^{(i, j)}, \cdots, x_{m}^{(i, j)}\right), & \text { if } i \text { is even } .\end{cases}
$$

For example,

$$
F^{\prime}(3,2)=\left(\begin{array}{cc|cc|cc}
x_{2}^{(1,1)} & x_{2}^{(1,1)} & x_{2}^{(1,2)} & x_{2}^{(1,2)} & x_{2}^{(1,3)} & x_{2}^{(1,3)} \\
x_{1}^{(1,1)} & x_{2}^{(1,1)} & x_{1}^{(1,2)} & x_{2}^{(1,2)} & x_{1}^{(1,3)} & x_{2}^{(1,3)} \\
\hline x_{1}^{(2,1)} & x_{2}^{(2,1)} & x_{1}^{(2,2)} & x_{2}^{(2,2)} & x_{1}^{(2,3)} & x_{2}^{(2,3)} \\
x_{2}^{(2,1)} & x_{2}^{(2,1)} & x_{2}^{(2,2)} & x_{2}^{(2,2)} & x_{2}^{(2,3)} & x_{2}^{(2,3)} \\
\hline x_{2}^{(3,1)} & x_{2}^{(3,1)} & x_{2}^{(3,2)} & x_{2}^{(3,2)} & x_{2}^{(3,3)} & x_{2}^{(3,3)} \\
x_{1}^{(3,1)} & x_{2}^{(3,1)} & x_{1}^{(3,2)} & x_{2}^{(3,2)} & x_{1}^{(3,3)} & x_{2}^{(3,3)}
\end{array}\right) .
$$


Theorem 3.4. Let $F^{\prime}(N, m)$ be a block hook matrix of order Nm defined as (29). Then

$$
\operatorname{det}\left(F^{\prime}(N, m)\right)= \begin{cases}(-1)^{\frac{N m}{4}} \prod_{i=1}^{m} \operatorname{det}\left(X_{(i, i+1)}\right), & \text { if } N \text { is even, } m \text { is even } \\ (-1)^{\frac{N(m-1)}{4}} \prod_{i=1}^{m} \operatorname{det}\left(X_{(i, i+1)}\right), & \text { if } N \text { is even, } m \text { is odd } \\ (-1)^{\frac{(N-1) m}{4}} \prod_{i=1}^{m} \operatorname{det}\left(X_{(i, i+1)}\right), & \text { if } N \text { is odd, } m \text { is even } \\ (-1)^{\frac{(N-1)(m-1)}{4}} \prod_{i=1}^{m} \operatorname{det}\left(X_{(i, i+1)}\right), & \text { if } N \text { is odd, } m \text { is odd }\end{cases}
$$

where $X_{(i, i+1)}$ is defined as (6) and $x_{m+1}^{(i, j)}=0$ for all $1 \leq i, j \leq N$.

Proof. In this case also we apply the same row operations described by (23) and (24) on the matrix $D(N, m)$, and we get the matrix $F^{\prime}(N, m)$. This completes the proof.

\section{Block matrices containing hooks of four different shapes}

In this section, we are going to derive the determinantal formulas of block hook matrices, in which the blocks are suitable combination of four different shapes of hooks. In particular, here we construct two such block matrices and show that the determinants of these matrices are also product of determinants of matrices $X_{(i, i+1)},(i=1,2, \cdots, m)$ (defined as (6)). So let us define a block hook matrix $G(N, m)$ as follows:

$$
G(N, m)=\left(G_{i j}\right)_{N \times N}, \text { where } G_{i j}= \begin{cases}B_{m}\left(x_{1}^{(i, j)}, \cdots, x_{m}^{(i, j)}\right), & \text { if } i, j \text { are odd } \\ C_{m}\left(x_{1}^{(i, j)}, \cdots, x_{m}^{(i, j)}\right), & \text { if } i \text { is odd, } j \text { is even } \\ D_{m}\left(x_{1}^{(i, j)}, \cdots, x_{m}^{(i, j)}\right), & \text { if } i \text { is even, } j \text { is odd } \\ A_{m}\left(x_{1}^{(i, j)}, \cdots, x_{m}^{(i, j)}\right), & \text { if } i, j \text { are even } .\end{cases}
$$

For example,

$$
G(3,2)=\left(\begin{array}{cc|cc|cc}
x_{1}^{(1,1)} & x_{2}^{(1,1)} & x_{2}^{(1,2)} & x_{1}^{(1,2)} & x_{1}^{(1,3)} & x_{2}^{(1,3)} \\
x_{2}^{(1,1)} & x_{2}^{(1,1)} & x_{2}^{(1,2)} & x_{2}^{(1,2)} & x_{2}^{(1,3)} & x_{2}^{(1,3)} \\
\hline x_{2}^{(2,1)} & x_{2}^{(2,1)} & x_{2}^{(2,2)} & x_{2}^{(2,2)} & x_{2}^{(2,3)} & x_{2}^{(2,3)} \\
x_{1}^{(2,1)} & x_{2}^{(2,1)} & x_{2}^{(2,2)} & x_{1}^{(2,2)} & x_{1}^{(2,3)} & x_{2}^{(2,3)} \\
\hline x_{1}^{(3,1)} & x_{2}^{(3,1)} & x_{2}^{(3,2)} & x_{1}^{(3,2)} & x_{1}^{(3,3)} & x_{2}^{(3,3)} \\
x_{2}^{(3,1)} & x_{2}^{(3,1)} & x_{2}^{(3,2)} & x_{2}^{(3,2)} & x_{2}^{(3,3)} & x_{2}^{(3,3)}
\end{array}\right) .
$$

Theorem 4.1. Let $G(N, m)$ be a block hook matrix of order $N m$ defined as (31). Then

$$
\operatorname{det}(G(N, m))=\prod_{i=1}^{m} \operatorname{det}\left(X_{(i, i+1)}\right)
$$

where $X_{(i, i+1)}$ is defined by (6) and $x_{m+1}^{(i, j)}=0$ for all $1 \leq i, j \leq N$.

Proof. Here we apply the same row and column operations described by (15) and (16) on $B(N, m)$. Clearly after the effect of these row and column operations we get the matrix $G(N, m)$. Consequently we get the result. 
Let us introduce one more block hook matrix $G^{\prime}(N, m)$ containing four different shape of hooks as follows:

$$
G^{\prime}(N, m)=\left(G_{i j}^{\prime}\right)_{N \times N}, \text { where } G_{i j}^{\prime}= \begin{cases}A_{m}\left(x_{1}^{(i, j)}, \cdots, x_{m}^{(i, j)}\right), & \text { if } i, j \text { are odd } \\ D_{m}\left(x_{1}^{(i, j)}, \cdots, x_{m}^{(i, j)}\right), & \text { if } i \text { is odd, } j \text { is even } \\ C_{m}\left(x_{1}^{(i, j)}, \cdots, x_{m}^{(i, j)}\right), & \text { if } i \text { is even }, j \text { is odd } \\ B_{m}\left(x_{1}^{(i, j)}, \cdots, x_{m}^{(i, j)}\right), & \text { if } i, j \text { are even } .\end{cases}
$$

For example,

$$
G^{\prime}(3,2)=\left(\begin{array}{cc|cc|cc}
x_{2}^{(1,1)} & x_{2}^{(1,1)} & x_{2}^{(1,2)} & x_{2}^{(1,2)} & x_{2}^{(1,3)} & x_{2}^{(1,3)} \\
x_{2}^{(1,1)} & x_{1}^{(1,1)} & x_{1}^{(1,2)} & x_{2}^{(1,2)} & x_{2}^{(1,3)} & x_{1}^{(1,3)} \\
\hline x_{2}^{(2,1)} & x_{1}^{(2,1)} & x_{1}^{(2,2)} & x_{2}^{(2,2)} & x_{2}^{(2,3)} & x_{1}^{(2,3)} \\
x_{2}^{(2,1)} & x_{2}^{(2,1)} & x_{2}^{(2,2)} & x_{2}^{(2,2)} & x_{2}^{(2,3)} & x_{2}^{(2,3)} \\
\hline x_{2}^{(3,1)} & x_{2}^{(3,1)} & x_{2}^{(3,2)} & x_{2}^{(3,2)} & x_{2}^{(3,3)} & x_{2}^{(3,3)} \\
x_{2}^{(3,1)} & x_{1}^{(3,1)} & x_{1}^{(3,2)} & x_{2}^{(3,2)} & x_{2}^{(3,3)} & x_{1}^{(3,3)}
\end{array}\right) .
$$

Theorem 4.2. Let $G^{\prime}(N, m)$ be a block hook matrix of order $N m$ defined as (33). Then

$$
\operatorname{det}\left(G^{\prime}(N, m)\right)=\prod_{i=1}^{m} \operatorname{det}\left(X_{(i, i+1)}\right)
$$

where $X_{(i, i+1)}$ is defined by (6) and $x_{m+1}^{(i, j)}=0$ for all $1 \leq i, j \leq N$.

Proof. Applying the same row and column operations depicted by (15) and (16) on the matrix $A(N, m)$, we get the matrix $G^{\prime}(N, m)$. Hence the theorem.

\section{Combinatorial interpretations of hook determinants}

Combinatorial interpretations of determinants can bring deeper understanding to their evaluations; this is especially true when the entries of a matrix have natural graph theoretic descriptions [2, 3, 5, 9, 15]. In this section, we give combinatorial interpretations of our main results (that are stated in previous sections) regarding hook determinants. Before plunging into the proof, let us recall the celebrated "Gessel-Lindström-Viennot" lemma. See [1, 5], for details. For the sake of completeness, let us reproduce the lemma from [1]. Let $\Gamma$ be a weighted, acyclic digraph. The vertex set and the edge set of the graph $\Gamma$, denoted by $V(\Gamma)$ and $E(\Gamma)$ respectively. A path in $\Gamma$ is a sequence of distinct vertices $v_{1}, v_{2}, \cdots, v_{r}$ such that $v_{i}, v_{i+1}(i=1, \cdots, r-1)$ is an edge directed from $v_{i}$ to $v_{i+1}$. For simplicity we denote a path $v_{1}, v_{2}, \cdots, v_{r}$ by $v_{1} v_{2} \cdots v_{r}$ and hence an edge $v_{i}, v_{i+1}$ by $v_{i} v_{i+1}$. The weight of a path $P$, denoted by $w(P)$ is the product of weights of all edges involved in the path and the length of a path $P$, denoted by $\ell(P)$, is the number of edges involved in the path $P$. Suppose that $U=\left\{u_{1}, u_{2}, \cdots, u_{n}\right\}$ and $V=\left\{v_{1}, v_{2}, \cdots, v_{n}\right\}$ are two $n$-sets of vertices of $\Gamma$ (not necessarily disjoint). To $U$ and $V$, associate the path matrix $M=\left(m_{i j}\right)_{n \times n}$, where $m_{i j}=\sum_{P: u_{i} \rightarrow v_{j}} w(P), P: u_{i} \rightarrow v_{j}$ denotes a path from $u_{i}$ to $v_{j}$. A path system from $U$ to $V$ is an ordered pair $(\mathcal{P}, \sigma)$, where $\sigma$ is a permutation of $n$ element set and $\mathcal{P}$ is a set of $n$ paths $P_{i}: u_{i} \rightarrow v_{\sigma(i)}$. The sign of a path system $(\mathcal{P}, \sigma)$ is $\operatorname{sgn}(\sigma)$. The weight of $(\mathcal{P}, \sigma)$, denoted by $w(\mathcal{P}, \sigma)$ is $\prod_{i=1}^{n} w\left(P_{i}\right)$. We call the path system vertex-disjoint if no two paths have a common vertex. Let $V D_{\Gamma}$ be the family of vertex-disjoint path systems in the graph $\Gamma$. Then the Gessel-Lindström-Viennot lemma is the following; 
Lemma 5.1 (Gessel-Lindström-Viennot lemma). Let $\Gamma=(V(\Gamma), E(\Gamma))$ be a weighted acyclic digraph. Suppose $U=\left\{u_{1}, u_{2}, \cdots, u_{n}\right\}, V=\left\{v_{1}, v_{2}, \cdots, v_{n}\right\}$ are two $n$-sets of vertices of $\Gamma$ (not necessarily disjoint) and $M$ be a path matrix from $U$ to $V$. Then

$$
\operatorname{det}(M)=\sum_{(\mathcal{P}, \sigma) \in V D_{\Gamma}} \operatorname{sgn}(\mathcal{P}, \sigma) w(\mathcal{P}, \sigma) .
$$

Now we give combinatorial proofs of theorems stated in previous sections using Lemma 5.1.

Combinatorial proof of Theorem 1.1. Consider the acyclic weighted digraph in Figure 2. We choose the sets $\left\{u_{1}, u_{2}, \cdots, u_{m}\right\}$ and $\left\{v_{1}, v_{2}, \cdots, v_{m}\right\}$ as the initial and terminal sets of vertices respectively. Clearly the associate path matrix is the matrix $A_{m}\left(x_{1}, \cdots, x_{m}\right)$ defined as (1). Evidently, there is only one vertex disjoint path system $(\mathcal{P}, \mathrm{Id})$, where Id is the identity permutation of $m$ element set and $\mathcal{P}$ is a set of $m$ paths $P_{i}: u_{i} \rightarrow$ $v_{i}(i=1, \cdots, m)$. Now applying Lemma 5.1 we get the theorem.
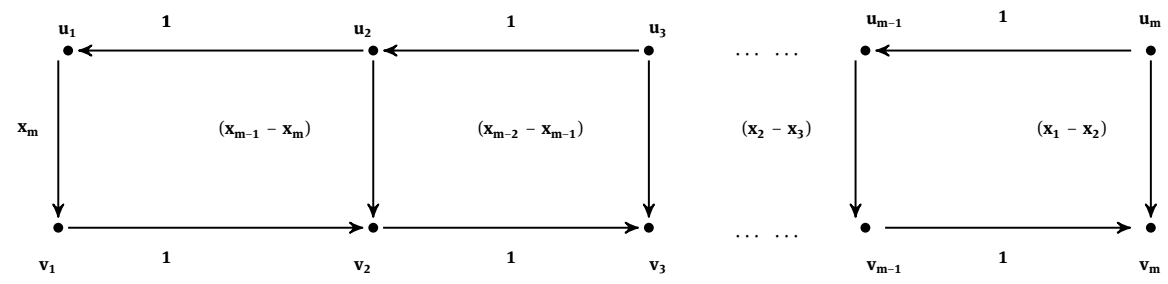

Figure 2: A weighted acyclic digraph $\Gamma_{m}$, and the weight of each edge is described on figure.

Remark 2. In exactly the same way we can give combinatorial explanation of theorems 1.2, 1.3 and 1.4. In those cases also we use $\Gamma_{m}$ as combinatorial object possibly permuting some of its vertices.

Combinatorial proof of Theorem 2.1. To give a combinatorial interpretation of the theorem we use GesselLindström-Viennot lemma. So first we have to construct a weighted acyclic digraph $\Gamma_{N, m}$ whose path matrix is the matrix $A(N, m)$ (defined as (7)). For each $i \in[m]$, let us define $\tilde{U}_{i}=\left\{u_{i}, u_{m+i}, \cdots, u_{(N-2) m+i}, u_{(N-1) m+i}\right\}$ and $\tilde{V}_{i}=\left\{v_{i}, v_{m+i}, \cdots, v_{(N-2) m+i}, v_{(N-1) m+i}\right\}$. We declare the vertex set $V\left(\Gamma_{N, m}\right)=\bigcup_{i \in[m]}\left(\tilde{U}_{i} \cup \tilde{V}_{i}\right)$. Now in $\Gamma_{N, m}$, we want the vertices $\tilde{U}_{i} \cup \tilde{V}_{i}$ to form a weighted complete bipartite digraph $\Gamma_{i} \cong K_{N, N}$ with bi-partition sets $\tilde{U}_{i}$ and $\tilde{V}_{i}$ for each $i \in[m]$. The direction of each edge $u_{k} v_{\ell}(k=t m+i, \ell=r m+i$, where $t, \ell \in$ $\{0, \cdots, N-1\})$ is always taken from $u_{k}$ towards $v_{\ell}$ and $w\left(u_{k} v_{\ell}\right)=x_{m-i+1}^{(t+1, r+1)}-x_{m-i+2}^{(t+1, r+1)}$. Clearly each $\Gamma_{i}$ is acyclic. Now we finish the construction of $\Gamma_{N, m}$ by adjoining additional weighted directed edges to $\cup_{i=1}^{m} E\left(\Gamma_{i}\right)$, as depicted in Figure 3 Notice that $\Gamma_{N, m}$ is acyclic. Now we call $U=\left\{u_{1}, u_{2}, \cdots, u_{N m}\right\}$ to be the initial set of vertices and $V=\left\{v_{1}, v_{2}, \cdots, v_{N m}\right\}$ to be the terminal set of vertices of the graph $\Gamma_{N, m}$. Then the path matrix of the graph $\Gamma_{N, m}$ is the matrix $A(N, m)$.

So, by Gessel-Lindström-Viennot lemma, we can write

$$
\operatorname{det}(A(N, m))=\sum_{\mathcal{P}_{\Gamma_{N, m}} \in V D_{\Gamma_{N, m}}} \operatorname{sgn}\left(\mathcal{P}_{\Gamma_{N, m}}\right) w\left(\mathcal{P}_{\Gamma_{N, m}}\right) .
$$

Now we have to characterize all the vertex disjoint path systems in the graph $\Gamma_{N, m}$. The following lemmas give the complete characterization of all vertex disjoint path systems in $\Gamma_{N, m}$.

Lemma 5.2. Let $\mathcal{P}_{\Gamma_{N, m}} \in V D_{\Gamma_{N, m}}$ and $P$ be a path in $\mathcal{P}_{\Gamma_{N, m}}$. Then $\ell(P)=1$.

Proof. Let $P$ be a path in $\mathcal{P}_{\Gamma_{N, m}}$ from the initial vertex $u_{\ell}$ to the terminal vertex $v_{k}$, for some $u_{\ell} \in U$ and $v_{k} \in V$. Then we show that the length of the path $P$ is 1 . In fact, length of $P>1$ implies, $P$ contains at least three distinct vertices. Then $P$ is of the form either $u_{\ell} u_{i} \cdots v_{k}$ or $u_{\ell} v_{j} \cdots v_{k}$, for some $u_{i} \in U$ and $v_{j} \in V$, 


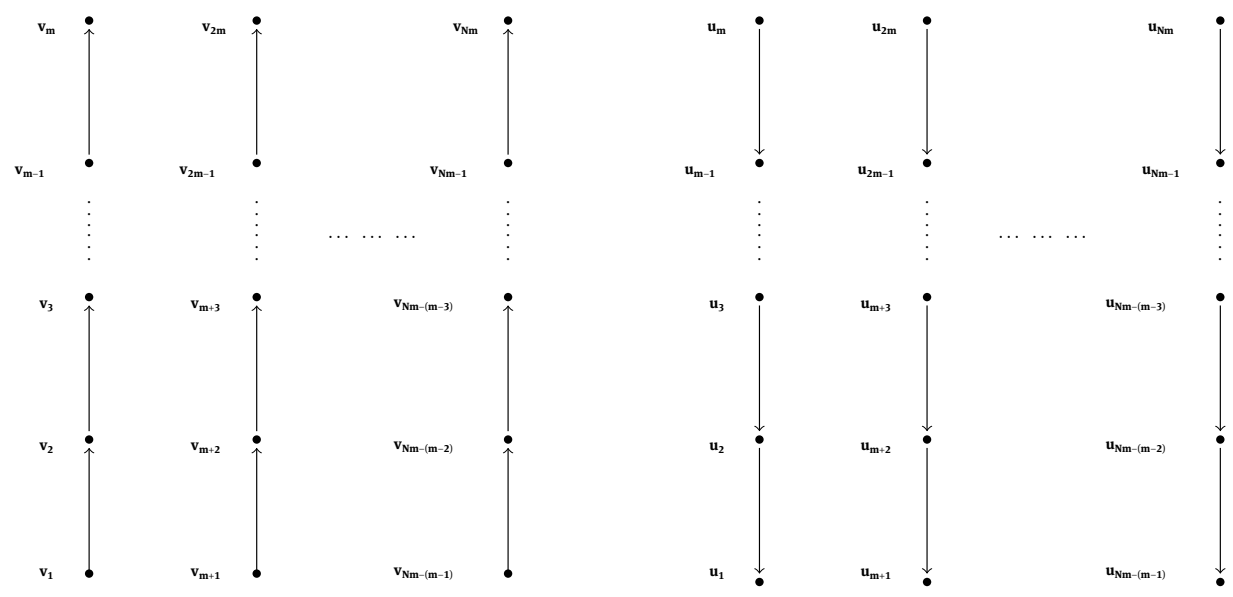

Figure 3: A weighted acyclic digraph with $w\left(u_{j+1} u_{j}\right)=1=w\left(v_{j} v_{j+1}\right)$, for $i=1, \cdots, N m-1$.

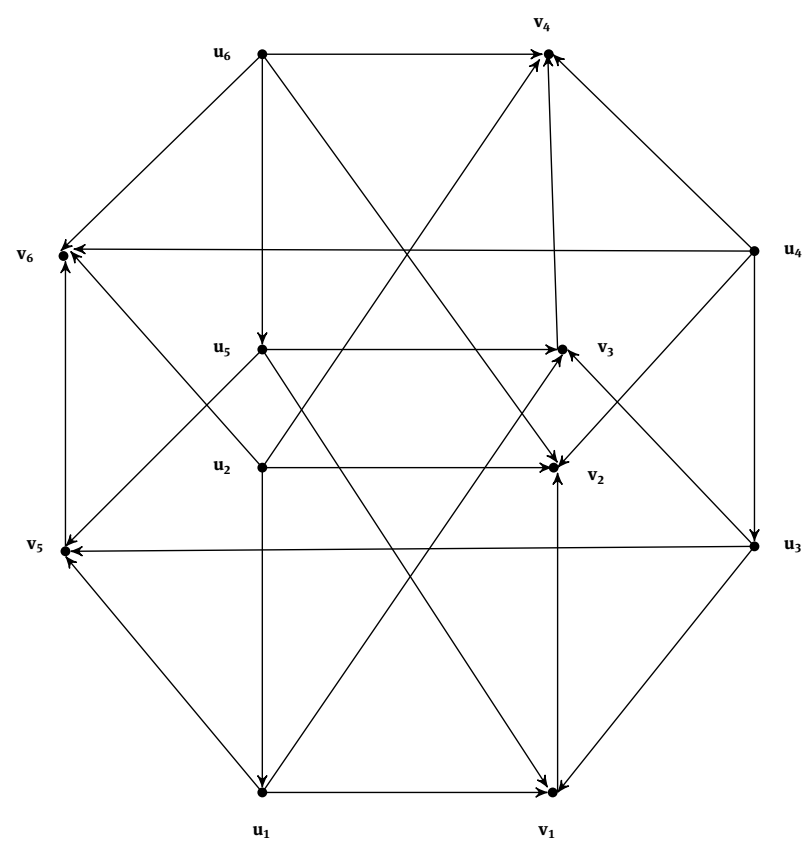

$$
\begin{aligned}
& w\left(u_{1} \mathbf{v}_{1}\right)=x_{2}^{(1,1)} \\
& w\left(u_{3} \mathbf{v}_{1}\right)=x_{2}^{(2,1)} \\
& w\left(u_{3} \mathbf{v}_{3}\right)=x_{2}^{(2,2)} \\
& w\left(\mathbf{u}_{5} \mathbf{v}_{3}\right)=x_{2}^{(3,2)} \\
& w\left(\mathbf{u}_{5} \mathbf{v}_{5}\right)=x_{2}^{(3,3)} \\
& w\left(\mathbf{u}_{1} \mathbf{v}_{5}\right)=x_{2}^{(1,3)} \\
& w\left(\mathbf{u}_{3} \mathbf{v}_{5}\right)=x_{2}^{(2,3)} \\
& w\left(\mathbf{u}_{1} \mathbf{v}_{3}\right)=x_{2}^{(1,2)} \\
& w\left(\mathbf{u}_{5} \mathbf{v}_{1}\right)=x_{2}^{(3,1)}
\end{aligned}
$$

$\mathbf{w}\left(\mathbf{u}_{6} \mathbf{u}_{5}\right)=\mathbf{w}\left(\mathbf{u}_{2} \mathbf{u}_{1}\right)=\mathbf{w}\left(\mathbf{u}_{4} \mathbf{u}_{3}\right)=\mathbf{1}$

$\mathbf{w}\left(\mathbf{v}_{1} \mathbf{v}_{2}\right)=\mathbf{w}\left(\mathbf{v}_{3} \mathbf{v}_{4}\right)=\mathbf{w}\left(\mathbf{v}_{5} \mathbf{v}_{6}\right)=\mathbf{1}$

Figure 4: A weighted acyclic digraph $\Gamma_{3,2}$. The weight of the edges appear on right side of this figure.

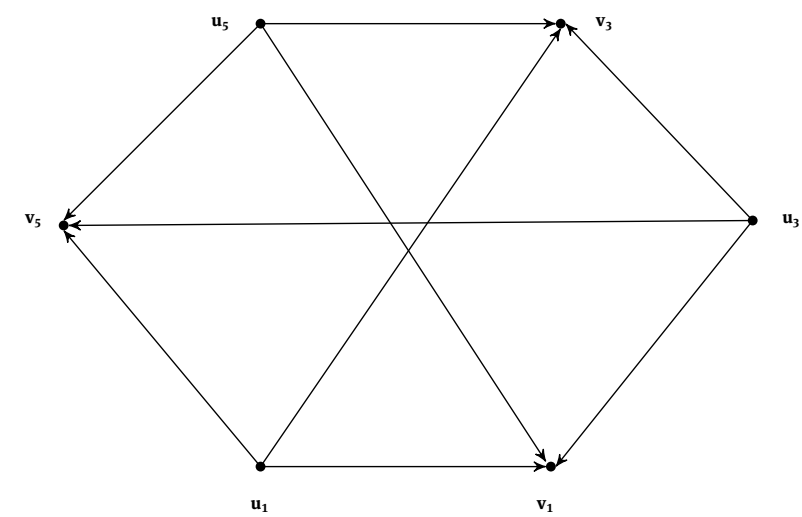

$$
\begin{aligned}
& w\left(\mathbf{u}_{1} \mathbf{v}_{1}\right)=x_{2}^{(1,1)} \\
& w\left(\mathbf{u}_{3} \mathbf{v}_{1}\right)=x_{2}^{(2,1)} \\
& w\left(\mathbf{u}_{3} \mathbf{v}_{3}\right)=x_{2}^{(2,2)} \\
& w\left(\mathbf{u}_{5} \mathbf{v}_{3}\right)=x_{2}^{(3,2)} \\
& w\left(\mathbf{u}_{5} \mathbf{v}_{5}\right)=x_{2}^{(3,3)} \\
& w\left(\mathbf{u}_{1} \mathbf{v}_{5}\right)=x_{2}^{(1,3)} \\
& w\left(\mathbf{u}_{3} \mathbf{v}_{5}\right)=x_{2}^{(2,3)} \\
& w\left(\mathbf{u}_{1} \mathbf{v}_{3}\right)=x_{2}^{(1,2)} \\
& w\left(\mathbf{u}_{5} \mathbf{v}_{1}\right)=x_{2}^{(3,1)}
\end{aligned}
$$

Figure 5: A weighted acyclic digraph $\Gamma_{1}$. The weight of the corresponding edges appear on figure. 

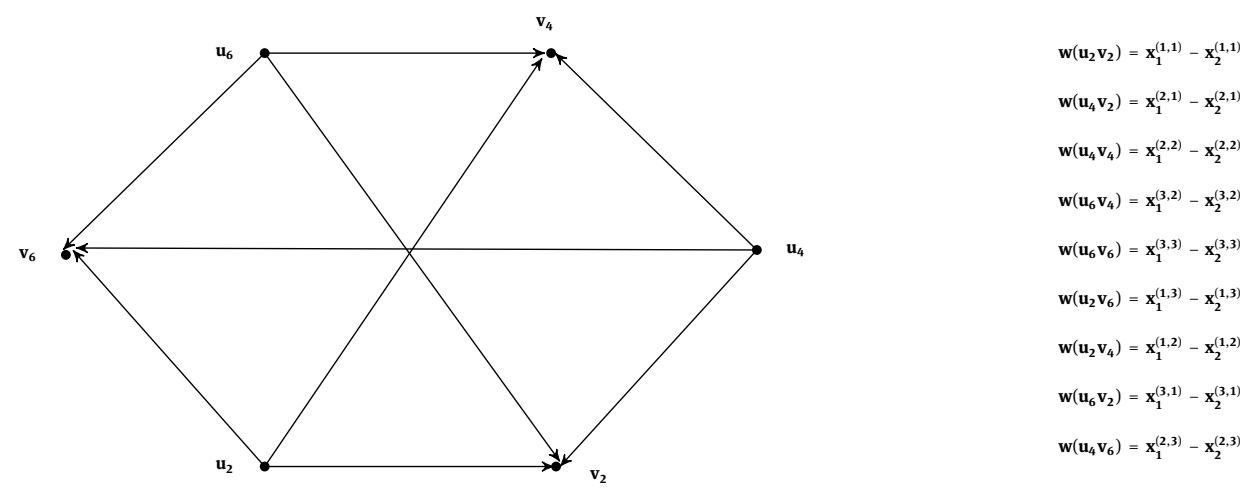

Figure 6: A weighted acyclic digraph $\Gamma_{2}$. The weight of the corresponding edges appear on figure.

i.e, the second vertex of $P$ (a vertex next to the initial vertex in $P$ ) is either $u_{i}$ or $v_{j}$. Suppose $P$ is $u_{\ell} u_{i} \cdots v_{k}$. Since $\mathcal{P}_{\Gamma_{N, m}}$ is a path system from $U$ to $V$, we must have another path $P$ in $\mathcal{P}_{\Gamma_{N, m}}$, whose initial vertex is $u_{i}$. This contradicts that the path system $\mathcal{P}_{\Gamma_{N, m}}$ is vertex disjoint. Again if $P$ is $u_{\ell} v_{j} \cdots v_{k}$, then in $\mathcal{P}_{\Gamma_{N, m}}$ we get another path $\tilde{P}$ whose terminal vertex is $v_{j}$, which also contradicts the fact $\mathcal{P}_{\Gamma_{N, m}}$ is vertex disjoint. Therefore, the length of each path in any vertex disjoint path system must be 1 .

In this portion we will think about the vertex disjoint path systems in subgraphs $\Gamma_{1}, \Gamma_{2}, \cdots, \Gamma_{m}$ of the graph $\Gamma_{N, m}$. For each $\Gamma_{i}(i=1, \cdots, m)$ we choose $\tilde{U}_{i}, \tilde{V}_{i}$ as the initial and terminal set of vertices respectively. It can be shown that the path matrix of the graph $\Gamma_{i}$ is the matrix $X_{(m-i+1, m-i+2)}$, defined as (6). From our construction of the graph $\Gamma_{N . m}$, it is evident that

$$
V D_{\Gamma_{N, m}}=\left\{(\mathcal{P}, \sigma)=\left(\mathcal{P}^{1} \cup \mathcal{P}^{2} \cup \cdots \cup \mathcal{P}^{m}, \sigma_{1} \sigma_{2} \cdots \sigma_{m}\right) \text {, where }\left(\mathcal{P}^{i}, \sigma_{i}\right) \text { is a path system of } \Gamma_{i}\right\} .
$$

Therefore, we can write

$$
\begin{aligned}
\sum_{(\mathcal{P}, \sigma) \in V D_{\Gamma_{N, m}}} \operatorname{sgn}(\mathcal{P}, \sigma) w(\mathcal{P}, \sigma)= \\
\quad\left(\sum_{\left(\mathcal{P}^{1}, \sigma_{1}\right) \in V D_{\Gamma_{1}}} \operatorname{sgn}\left(\mathcal{P}^{1}, \sigma_{1}\right) w\left(\mathcal{P}^{1}, \sigma_{1}\right)\right) \cdots\left(\sum_{\left(\mathcal{P}^{m}, \sigma_{m}\right) \in V D_{\Gamma_{m}}} \operatorname{sgn}\left(\mathcal{P}^{m}, \sigma_{m}\right) w\left(\mathcal{P}^{m}, \sigma_{m}\right)\right) .
\end{aligned}
$$

Now, $\left(\sum_{\left(\mathcal{P}^{i}, \sigma_{i}\right) \in V D_{\Gamma_{i}}} \operatorname{sgn}\left(\mathcal{P}^{i}, \sigma_{i}\right) w\left(\mathcal{P}^{i}, \sigma_{i}\right)\right)=\operatorname{det}\left(X_{(m-i+1, m-i+2)}\right)$, where $i \in[m]$ and $X_{(m-i+1, m-i+2)}$ is the path matrix associated to the graph $\Gamma_{i}$. Hence the theorem.

Example 5.3. If we take $N=3, m=2$, then the weighted acyclic digraph $\Gamma_{3,2}$ is Figure 4 and $\Gamma_{1}, \Gamma_{2}$ are Figures 5, 6 respectively. Moreover the path matrix associated to $\Gamma_{3,2}$ is $A(3,2)$ defined as (8). Consider a vertex disjoint path system $u_{1} v_{1}, u_{2} v_{2}, \cdots, u_{6} v_{6}$ in $\Gamma$, where each path $u_{i} v_{i}(i=1, \cdots, 6)$ is an edge. Notice that, three paths $u_{1} v_{1}, u_{3} v_{3}, u_{5} v_{5}$ from the above path system is a vertex disjoint path system in $\Gamma_{1}$ and three another paths $u_{2} v_{2}, u_{4} v_{4}, u_{6} v_{6}$ is a vertex disjoint path system in $\Gamma_{2}$. Again $u_{1} v_{5}, u_{2} v_{2}, u_{3} v_{1}, u_{4} v_{6}, u_{5} v_{3}, u_{6} v_{4}$ is vertex disjoint path system in $\Gamma$. For this vertex disjoint path system, $u_{1} v_{5}, u_{5} v_{3}, u_{3} v_{1}$ is a vertex disjoint path system in $\Gamma_{1}$ and $u_{2} v_{2}, u_{4} v_{6}, u_{6} v_{4}$ is a vertex disjoint path system in $\Gamma_{2}$.

Remark 3. In exactly the same way we can give combinatorial explanation of all other theorems. In those cases also we use $\Gamma_{N, m}$ as combinatorial object possibly permuting some of its vertices.

Acknowledgment: I would like to thank my mentor Prof. Arvind Ayyer for giving this problem, insightful discussion to solve the problem and valuable suggestions in the preparation of this paper. Also I would like to thank Prof. Darij Grinberg for his helpful suggestions in the preparation of this paper. The author 
was supported by Department of Science and Technology grant EMR/2016/006624 and partly supported by UGC Centre for Advanced Studies. Also the author was supported by NBHM Post Doctoral Fellowship grant 0204/52/2019/RD-II/339. The author would like to thank the anonymous referee for meticulous reading of the manuscript and valuable suggestions that significantly improved the exposition of this paper.

Data Availability Statement: Data sharing is not applicable to this article as no datasets were generated or analyzed during the current study.

\section{References}

[1] M. Aigner, A course in enumeration, Graduate Texts in Mathematics, vol. 238, Springer, Berlin, 2007.

[2] A. Ayyer, Determinants and perfect matchings, J. Combin. Theory Ser. A 120 (2013), no. 1, 304-314.

[3] S. Bera and S. K. Mukherjee, Combinatorial proofs of some determinantal identities, Linear Multilinear Algebra 66 (2018), no. 8, 1659-1667.

[4] G. Bhatnagar and C. Krattenthaler, Spiral determinants, Linear Algebra Appl. 529 (2017), 374-390.

[5] I. Gessel and G. Viennot, Binomial determinants, paths, and hook length formulae, Adv. in Math. 58 (1985), no. 3, 300-321.

[6] C. Krattenthaler, Advanced determinant calculus, Sém. Lothar. Combin. 42 (1999), Art. B42q, 67.

[7] __ Advanced determinant calculus: a complement, Linear Algebra Appl. 411 (2005), 68-166.

[8] L. G. Molinari, Determinants of block tridiagonal matrices, Linear Algebra Appl. 429 (2008), no. 8-9, 2221-2226.

[9] S. K. Mukherjee and S. Bera, Combinatorial proofs of the Newton-Girard and Chapman-Costas-Santos identities, Discrete Math. 342 (2019), no. 6, 1577-1580.

[10] E. M. Murman and S. S. Abarbanel, Progress and supercomputing in computational fluid dynamics, Proceedings of U. S.Israel Workshop, Birkhäuser Boston, Inc., Germany, 1984.

[11] O. Popescu, C. Rose, and D. C. Popescu, Maximizing the determinant for a special class of block-partitioned matrices, Math. Probl. Eng. (2004), no. 1, 49-61.

[12] P. D. Powell, Calculatting determinants of block matrices, arXiV:1112.4379v1 [math.RA] (2011).

[13] C. Roessner, S. Ratti and W. Weise, Polyakov loop, diquarks and the two-flavour phase diagram, Phys. Rev. D. 75 (2007), 034007-034004.

[14] B. Sasaki, C. Friman and K. Redlich, Susceptibilities and the phase structure of a chiral model with polyakov loops, Phys. Rev. D. 75 (2007), 074013-074029.

[15] D. Zeilberger, A combinatorial proof of Newton's identities, Discrete Math. 49 (1984), 319.

[16] D. Zheng, Matrix methods for determinants of Pascal-like matrices, Linear Algebra Appl. 577 (2019), 94-113. 